\title{
Resolving geometrically necessary dislocation density onto individual dislocation types using EBSD-based continuum dislocation microscopy
}

\author{
T.J. Ruggles ${ }^{\mathrm{a}, \mathrm{b}, *}$, D.T. Fullwood ${ }^{\mathrm{b}}$, J.W. Kysar ${ }^{\mathrm{c}}$ \\ ${ }^{a}$ National Institute of Aerospace, 100 Exploration Way, Hampton, VA 23666, USA \\ ${ }^{b}$ Department of Mechanical Engineering, Brigham Young University, Provo, UT 84602, United States \\ ${ }^{c}$ Department of Mechanical Engineering, Columbia University, New York, NY 10027, United States
}

\begin{abstract}
Modeling of plasticity is often hampered by the difficulty in accurately characterizing dislocation density on the microscale for real samples. It is particularly difficult to resolve measured dislocation content onto individual dislocation types at the length scales most commonly of interest in plasticity studies. Methods have been developed to analyze dislocation content at the continuum level using the Nye tensor and Kröner's fundamental relation of continuum dislocation theory to interpret the local strain gradients obtained from high resolution electron backscatter diffraction (HREBSD). This paper assesses an implementation of the NyeKröner-Bilby method for resolving measured geometrically necessary dislocation content onto individual dislocation types via a novel simulation of distortion fields around continuum fields of dislocation density based on classical continuum elasticity equations. It is then applied to HREBSD data for a micro-indented nickel single crystal.
\end{abstract}

Keywords: EBSD microscopy, dislocations, crack tip plasticity

\section{Introduction}

The motion, nucleation, and interaction of dislocations are key physical phenomena behind crystal plasticity. Partially as a result of the difficulty of accurately measuring dislocation content on individual slip systems, most plasticity models do not address the phenomenon directly; i.e. dislocation activity and content is not typically explicitly quantified, although such models are beginning to see development, e.g., (Evers et al., 2004; Ma et al., 2006; Baitsch et al., 2015). Electron backscatter diffraction (EBSD) and X-ray diffraction techniques (Ungár et al., 1998; Field et al., 2010; Lim et al., 2014; Jiang et al., 2015; Sun et al., 2000; El-Dasher et al., 2003; Pantleon, 2008; Ruggles and Fullwood, 2013; Zhang et al., 2014) have been developed to provide local estimates of bulk geometrically necessary dislocation (GND) density (defined in terms of the line length of dislocations in a unit volume, not including dipoles present within the measurement volume). However, these methods generally do not directly determine the relative activity of dislocations of different dislocation types, the critical information required for a detailed physical model.

Because dislocations exist on the scale of the crystal lattice, full characterization of dislocation type and structure has generally remained the realm of transmission electron microscopy (TEM) (e.g. Hirsch et al. (1960)), a process made impractical for meso-scale quantitative analysis by meticulous sample preparation

${ }^{*}$ Corresponding author. Tel.: +1 8032709984

Email address: timmyruggs@gmail . com (T.J. Ruggles) 
and by the need to examine very small volumes. The resolution and sample length scales of modern EBSD techniques, however, provide the potential for mapping relative dislocation activity at the length scales of interest in strain gradient crystal plasticity models, i.e. from a few hundred nanometers to tens of microns (Bardella and Giacomini, 2008; Fleck and Hutchinson, 1997). The recent advent of High Resolution EBSD (HREBSD) (Troost et al., 1993; Wilkinson et al., 2006a,b; Kacher et al., 2009) allows for the accurate determination of local strain gradients, combined with improved spatial and angular resolution, thus shedding new light on dislocation content. Reviews of EBSD-based continuum dislocation microscopy and HREBSD techniques are provided in the literature (Gardner et al., 2010; Wilkinson et al., 2010). Once local deformation gradients are determined via EBSD or HREBSD techniques, they may be related to the geometrically necessary dislocation content of the material via classical equations developed by Nye and Kröner in the 1950s. This paper refers to the extraction of dislocation information from EBSD data via these equations as the Nye-Kröner-Bilby method.

Although the Nye-Kröner-Bilby method theoretically allows for the determination of dislocation density for each dislocation type, actual determination of these densities is problematic because of the underconstrained nature of the Nye-Kröner-Bilby equations and the 2D nature of most EBSD techniques. Care must be taken when applying the method because results can be highly sensitive to specific details of implementation. Because of this, most researchers do not report dislocation densities on individual slip systems, with some exceptions (Kysar et al., 2007, 2010; Britton and Wilkinson, 2012). Kysar et al. deal with this ambiguity by examining a single crystal with a precise orientation that has been deformed in plane strain. Knowledge of the sample's deformation allows for a number of additional constraints to be implemented alongside the Nye-Kröner-Bilby equations, bypassing their ambiguity. Using this highly constrained implementation of the Nye-Kröner-Bilby equations, Kysar achieves good agreement between measured dislocation density and plasticity simulations (Kysar et al., 2007, 2010; Saito et al., 2012), but its use is limited to a very specific deformation case. This paper assesses a general implementation of the Nye-Kröner-Bilby method that requires no a priori knowledge of the deformation of the specimen and makes no assumptions about which slip systems are active. This implementation is first evaluated using simulated distortion fields (Ruggles and Fullwood, 2013), and then compared to the well studied test case from Kysar et al. (2010). The general implementation fits both the simulations and past data extremely well while at the same time being generally applicable to any orientation or deformation mode, capturing more out of plane deformation, and recovering more degrees of freedom concerning the active slip systems.

\section{Nye-Kröner-Bilby Continuum Dislocation Theory}

EBSD dislocation microscopy relies on fundamental relationships between residual elastic deformation and continuum dislocation density fields developed in the 1950s by Nye, Kröner and Bilby. It is typical in EBSD dislocation microscopy to simplify Nye's relationship by assuming that all dislocation activity can be represented as superpositions of pure edge and screw dislocation types, as is typical for continuum dislocation studies. For example, an FCC material has 12 unique slip systems, combinations of slip direction and slip plane. If we assume that dislocations associated with these slip systems can be either pure edge or pure screw, then that suggests 24 unique dislocation types. However, because screw dislocations have the same dislocation line vector regardless of their slip plane, each screw dislocation may belong to one of two slip systems, meaning that there are effectively only 18 unique dislocation types, 12 edges and 6 screws (Evers et al., 2004). Dislocations of opposite sign may be accommodated by considering positive and negative dislocation densities for each type. Making these simplifications, we state Nye's relationship 
as (Nye, 1953):

$$
\alpha=\sum_{t=1}^{N} \rho^{t} \mathbf{b}^{t} \otimes \mathbf{r}^{t}
$$

where $\alpha$ is known as the Nye tensor. This tensor provides a concise representation of net dislocation density and type on a continuum level. The other terms in Equation $1, \rho^{t}, \mathbf{b}^{t}$, and $\mathbf{r}^{t}$, represent the dislocation density, Burgers vector and dislocation line vector of each dislocation type (indicated by $t$ ), respectively. Kröner and Bilby then showed that the Nye tensor is related to the elastic distortion of the material as follows (Kröner, 1958; Bilby et al., 1958):

$$
\alpha=\nabla \times \beta
$$

where $\beta$ is the elastic lattice distortion tensor (measurable via HREBSD techniques). Equation 2 is referred to as the fundamental relation of continuum dislocation theory.

A natural approach to the extraction of relative GND content on individual dislocation types is to obtain the available components of the Nye tensor from Equation 2 (based upon measured values of $\beta$ ), and then extract the densities for individual dislocation types, $\rho^{t}$, from Equation 1. However, the Nye tensor has at most nine components (generally fewer than nine can actually be resolved from the EBSD measurements), whereas most materials (simple cubic materials being an exception) have significantly more unique dislocation types. Thus, Equation 1 is underconstrained when solving for $\rho^{t}$ based upon a known Nye tensor. Additional constraints, typically incorporated into some form of optimization approach, must be employed to find a reasonable estimate of dislocation density (Sun et al., 2000; El-Dasher et al., 2003). The two most common methods involve minimizing the $L_{2}$ norm (the vector 2-norm) of the individual dislocation densities, or the $L_{1}$ norm (the vector 1-norm) of the dislocation densities (Sun et al., 2000; Arsenlis and Parks, 1999). The $L_{2}$ method, while having no explicit physical meaning, is very simple to calculate using the pseudo-inverse to determine the least squares solution; the $L_{1}$ method is based upon minimizing total dislocation line length, and therefore total slip.

This paper minimizes the $L_{1}$ norm of the dislocation density solution vector to constrain the Nye-KrönerBilby relationships. This method is assumed to be more physically significant because the strain energy of dislocations is often assumed to be proportional to their length, as predicted by classical continuum elastic dislocation mechanics. Since dislocation distortion fields can interact, the linear assumption is not necessarily accurate (Wheeler et al., 2009), especially at higher dislocation densities. However, in this study, these interactions are assumed to be negligible, as is typically assumed in the literature. The individual dislocation densities in the $L_{1}$ norm may also be weighted according to Schmid factor and/or differences in the critical resolved shear stresses (CRSS) of each type in order to better reflect the propensity for a given system to be activated (Kysar et al., 2010) (for example, in magnesium the CRSS of pyramidal systems is many times higher than that of basal systems (Koike et al., 2003)). Edge and scew dislocations have different line energies, which may also be used to weight the solution (Wilkinson and Randman, 2010). If there are multiple Burgers vectors in a crystal system, their magnitudes can also be used to help determine appropriate weighting, as the magnitude of the Burgers vector is a major factor in the energy of a dislocation. Once an energy function is established, we may select a set of densities for each dislocation type, $\rho^{t}$, that minimizes this energy while still adhering to the constraint that these dislocation densities be consistent with the measured Nye tensor as per Equation 1. This problem may be formally stated as:

$$
\operatorname{minimize} \sum_{t=1}^{N}\left|w^{t} \rho^{t}\right| \text {, such that } \alpha=\sum_{t=1}^{N} \rho^{t} \mathbf{b}^{t} \otimes \mathbf{r}^{t}
$$

where $w^{t}$ is a weighting factor for each dislocation type that allows more energetically favorable dislocations 
to be better represented and $\alpha$ is calculated from measured EBSD data via Equation 2. In this study, weight factors are set to unity. For the FCC materials studied in this paper, Equation 3 represents an optimization problem with 18 unknowns ( $\rho^{t}$, the dislocation densities for each of the dislocation types) and up to nine linear constraints from the nine components of the Nye tensor. The objective function is proportional to the energy of the dislocation network being examined. The choice of minimization algorithm and algorithm starting point are non-trivial.

While Equation 3 is formulated in order to find the most physically likely set of $\rho^{t}$ in a material that can accommodate the measured residual distortion, the equation is more often used as a means of estimating bulk dislocation density (the sum of the individual densities) than as a method of determining accurate values for the individual $\rho^{t}$ (with some exceptions: (Karamched and Wilkinson, 2011; Britton and Wilkinson, 2012)). This is at least partially an acknowledgment that only a fraction of the required information is available and the difficulty in finding a global minimum for Equation 3; this will be apparent once the information available from EBSD is reviewed in the next section. When dislocation density is reported for various dislocation types, it is typically for a highly constrained case (Kysar et al., 2007, 2010), or to calculate relative amounts of non-basal slip in hexagonal materials (Littlewood et al., 2011; Britton et al., 2010; Khosravani et al., 2013). The difficulty in calculating relative activity has led many researchers to find estimates of the overall bulk dislocation density instead of trying to resolve the full set of $\rho^{t}$. The entrywise vector 1-norm of $\alpha$ (estimated from its measurable elements only) and kernel average misorientation have been used to find reasonable estimates of bulk dislocation density (El-Dasher et al., 2003; Ruggles and Fullwood, 2013; Calcagnotto et al., 2010; Kubin and Mortenson, 2003).

It should be emphasized that these relationships address only geometrically necessary dislocations associated with distortion gradients. Dislocations associated with homogeneous deformation that have no far field distortion effect are considered statistically stored dislocations (SSDs) and are not detectable via geometric measurement-based methods (Ashby, 1970). It should also be noted that the distortion fields of SSDs are only guaranteed to have no net geometric contribution at large length scales. At smaller length scales, SSDs may have a net geometric contribution (indeed, SSDs are physically identical to GNDs, it is only their distribution that distinguishes them). The size of the implicit Burgers circuit, within which the net dislocation content is quantified, is given by the step size used to determine the distortion gradients in the sample. Hence it is clear that the definition of measured GND content is length scale dependent and should not be quoted as though it were independent of measurement approach. The effect of the selection of step size on measured dislocation density is not discussed further in this work; the step size guidelines laid out by Kysar et al. are employed (Kysar et al., 2010). For the remainder of this paper, all references to dislocation density should be understood to mean geometrically necessary dislocation density.

\section{Methods}

\subsection{Distortion Data from HREBSD}

Adams and Sun et al. established the relationship between information recovered from EBSD and dislocation content as input into the Nye-Kröner-Bilby relations (Adams, 1997; Sun et al., 2000). Conventional EBSD methods, which are widely commercially available, determine the local phase and orientation of a crystalline material. It is most useful to denote this orientation in terms of $g$, the orientation matrix. Local variations in $g$ may be related to the lattice distortion tensor, $\beta$, using the small angle approximation, as follows:

$$
g \approx I+\omega
$$


where $\omega$ is the lattice rotation tensor and $I$ is the identity matrix. The elastic lattice distortion is given by

$$
\beta \approx \omega+\varepsilon
$$

where $\varepsilon$ is the elastic strain tensor. If the elastic strain is negligible, then

$$
\beta \approx \omega \approx g-I
$$

This approximation is commonly accepted as the basis for determination of Nye tensor components in Equation 2. Furthermore, the development of HREBSD has made the complete lattice distortion tensor (rotation and strain) available. Measurement of absolute elastic lattice distortion requires the use of simulated, strain free EBSD patterns and improved pattern center techniques (Kacher et al., 2009; Winkelmann, 2007; Britton et al., 2010; Basinger et al., 2011; Maurice et al., 2011; Fullwood et al., 2015), but for recovery of the Nye tensor via Equation 2, only derivatives of distortion are required. These can be accurately provided via HREBSD convolution methods that determine local shifts in electron backscatter patterns from neighboring points (Wilkinson et al., 2006a,b; Gardner et al., 2010). HREBSD accomplishes this by measuring shifts between regions of interest on related patterns via cross correlation techniques, and subsequently relating these shifts to 8 of the 9 degrees of freedom of the relative deformation tensor between the lattices. The ninth degree of freedom, the difference in circular strain, is recovered by assuming the surface is traction free, an assumption first made by Wilkinson and well validated by modeling (Hardin et al., 2015). This allows neighboring points to be directly compared to calculate lattice distortion gradients via the forward difference derivative:

$$
\left.\frac{d \beta}{d x_{i}}\right]_{\vec{p}}=\frac{\beta^{\vec{p}, \vec{p}+\Delta \vec{x}_{i}}}{L}
$$

where $\vec{p}$ is a location on the surface of a scan, $\Delta \vec{x}_{i}$ is the vector between the first pattern and an adjacent pattern, $L$ is the spacing between raster points and the magnitude of $\Delta \vec{x}_{i}$, and $\beta \vec{p}, \vec{p}+\Delta \vec{x}_{i}$ is the relative distortion between patterns at location $\vec{p}$ and $\vec{p}+\Delta \vec{x}_{i}$.

EBSD is fundamentally a surface characterization technique, resulting in known distortion derivatives in only two dimensions. Serial sectioning, either mechanical or via focused ion beam milling, may be used to examine multiple planes in order to take derivatives in the sample normal direction (e.g., Calcagnotto et al. (2010); Demir et al. (2009)), but generally these methods are expensive, have poor resolution, are time-consuming, and destroy the sample, making in situ tests impossible (Schwartz et al., 2009). For conventional surface EBSD, only 12 of the 18 necessary distortion derivatives required by Equation 2 can be readily obtained; subsequently only the right column of $\alpha$ (the $\alpha_{i 3}$ components) can be fully recovered to be used as constraints in Equation 3, with partial recovery of the other tensor components. Wheeler et al. point out that these components of the Nye tensor $\left(\alpha_{i 3}\right)$ represent the net Burgers vector for a circuit in the sample plane, and allow the extraction of some out-of-plane deformation information if additional constraints are imposed (Wheeler et al., 2009). If strain is neglected, then two additional $\alpha$ components are available, as well as one difference between two components (Pantleon, 2008). The available components of $\alpha$ from surface HREBSD techniques are illustrated by expanding Equation 2 in terms of the strain $(\varepsilon)$ and rotation $(\omega)$ terms of $\beta$, as seen in Equation 8. Strain and rotation are separated from distortion assuming infinitesimal strain, meaning that $\varepsilon$ and $\omega$ are the symmetric and antisymmetric parts of $\beta$, respectively.

$$
\alpha=\left[\begin{array}{ccc}
\tilde{\varepsilon}_{12,3}+\tilde{\omega}_{12,3}-\varepsilon_{13,2}-\omega_{13,2} & \varepsilon_{13,1}+\omega_{13,1}-\tilde{\varepsilon}_{11,3} & \varepsilon_{11,2}-\varepsilon_{12,1}-\omega_{12,1} \\
\tilde{\varepsilon}_{22,3}-\varepsilon_{23,2}-\omega_{23,2} & \varepsilon_{23,1}+\omega_{23,1}-\tilde{\varepsilon}_{21,3}-\tilde{\omega}_{21,3} & \varepsilon_{21,2}+\omega_{21,2}-\varepsilon_{22,1} \\
\tilde{\varepsilon}_{32,3}+\tilde{\omega}_{32,3}-\varepsilon_{33,2} & \varepsilon_{33,1}-\tilde{\varepsilon}_{31,3}-\tilde{\omega}_{31,3} & \varepsilon_{31,2}+\omega_{31,2}-\varepsilon_{32,1}-\omega_{32,1}
\end{array}\right]
$$


Unavailable derivatives of terms of the lattice rotation and strain tensors (i.e. derivatives in the direction normal to the surface) are marked with a tilde. Since diagonal components of the rotation tensor are zero, there are 30 strain and rotation terms remaining in the expanded representation. The missing $\alpha$ components limit the number of constraints available in Equation 3, and further complicate the recovery of relative dislocation activity. According to Equation 8, only 3 components of the Nye tensor (and thus 3 constraints for Equation 3) are obtainable in 2D HREBSD. However, if strain is assumed to be negligible (as is assumed in Nye's original formulation), then there are actually two additional components and one difference of components available, namely $\alpha_{12}$ and $\alpha_{21}$ and the difference between $\alpha_{11}$ and $\alpha_{22}$ (Pantleon, 2008). This 6-constraint implementation utilizes a total of eight non-zero rotation terms from Equation 8, whereas the 3-constraint implementation only uses four. This paper applies both the 6-constraint and 3-constraint implementations on Equation 3 to simulated distortion fields of continuum dislocation fields and real data to examine the effects of the additional constraints.

For the assumed FCC material, Equation 3 represents an optimization problem that may be implemented with the 3 or 6 linear constraints and 18 unknowns (as stated previously, FCC materials have 18 unique dislocation types). On the surface, Equation 3 appears to be a simple problem because the objective function and constraints are all linear. Such a simple optimization problem with linear equality constraints may be solved using the method of Lagrange multipliers. However, the piece-wise nature of the absolute value function in the objective function mean that there are a large number of potential local minima $\left(2^{N}\right.$, where $N$ is the number of dislocation types), making direct calculation impractical and gradient based optimization problematic because of high sensitivity to the starting point of the optimization algorithm. Previously, we stated that we treat dislocations of opposite sign as positive and negative densities of the same dislocation type. Instead, we repose the problem by treating positive and negative dislocations of the same type as completely separate dislocation types. This doubles the number of variables, but the variables are now all linear and strictly positive, so the problem may be solved as a linear programming problem as in Britton et al. (2010). This type of problem is much easier to solve with commonly available algorithms. In this work we use the active set algorithm from MATLAB, a quasi-Newton type algorithm. Simplex optimization and interior point methods, while computationally slightly faster, occasionally failed to converge. For convenience in depicting the solutions, we recombine the densities of the dislocations types with opposite sign into a single dislocation density that may be positive or negative.

\subsection{A Continuum Relationship between Dislocations and Distortion}

The accuracy of the 3 and 6 constraint implementations of the Nye-Kröner-Bilby method for resolving GND content, as described in the previous section onto different dislocation types are assessed, in part, using simulations of distortion gradients resulting from GND fields. These simulations are based on classical continuum elastic mechanics equations for fields around dislocations and have been previously utilized for the purpose of validating bulk dislocation density characterization techniques (Ruggles and Fullwood, 2013). The analytic relations between dislocation types and elastic distortion are well-suited for numerical calculation purposes, but are formulated for discrete dislocations and lead to zero curl of the distortion (and hence, a null Nye tensor; Equation 2) at all locations except at the dislocation core, which is a singularity. In order to apply the analytic model to a continuum-level dislocation field, discrete dislocations are "smeared" over the simulated region of interest, i.e. they are modeled as if individual dislocations were comprised of an infinite number of dislocations with infinitesimally small Burgers vectors giving the same net Burgers vector. The resulting field may be readily scaled by a density. This method is more computationally efficient than standard Green's function techniques (Mura, 1963, 1982; Teodosiu, 1982; Hardin et al., 2013). For the reader's convenience, the development of these simulations is duplicated here from a previous work by the authors (Ruggles and Fullwood, 2013). 
Assuming that the $\mathrm{z}$ direction lies along the dislocation line, the $\mathrm{x}$ direction is in the direction of the Burgers vector and the y direction is perpendicular to the slip plane, the analytic equations for the non-zero distortion components around an edge dislocation in a linear elastic isotropic medium are (Lazar, 2003):

$$
\begin{array}{r}
\beta_{x x}=-\frac{b}{4 \pi(1-v)} \frac{y}{r^{2}}\left((1-2 v)+\frac{2 x^{2}}{r^{2}}\right) \text { where } r^{2}=x^{2}+y^{2} \\
\beta_{x y}=\frac{b}{4 \pi(1-v)} \frac{x}{r^{2}}\left((3-2 v)-\frac{2 y^{2}}{r^{2}}\right) \\
\beta_{y x}=-\frac{b}{4 \pi(1-v)} \frac{x}{r^{2}}\left((1-2 v)+\frac{2 y^{2}}{r^{2}}\right) \\
\beta_{y y}=-\frac{b}{4 \pi(1-v)} \frac{y}{r^{2}}\left((1-2 v)-\frac{2 x^{2}}{r^{2}}\right)
\end{array}
$$

where $b$ is the magnitude of Burgers vector and $v$ is Poisson's ratio. For a discrete screw dislocation (with line vector in the $\mathrm{z}$ direction), the distortions are given by:

$$
\beta_{z x}=-\frac{b}{2 \pi} \frac{y}{r^{2}}, \beta_{z y}=-\frac{b}{2 \pi} \frac{x}{r^{2}}
$$

If a continuous field of edge dislocations is assumed, with line directions in the $z$-direction, that occupies an infinite rectangular column between $x=s$ and $x=t, y=p$ and $y=q$, then the resultant $\beta_{x x}$ distortion (for example) at a point $x_{0}, y_{0}$ (for any $z$ ) is given by:

$$
\begin{array}{r}
\beta_{x x}\left(x_{0}, y_{0}\right)=-\frac{b}{4 \pi(1-v)(t-s)(q-p)} \int_{y=p x=s}^{q} \int_{x}^{t} \frac{y_{0}-y}{r_{0}^{2}}\left((1-2 v)+\frac{2\left(x_{0}-x\right)^{2}}{r_{0}^{2}}\right) d x d y \\
\quad \text { where } r_{0}^{2}=\left(x_{0}-x\right)^{2}+\left(y_{0}-y\right)^{2}
\end{array}
$$

Full solutions for the distortion field of a smeared edge and screw dislocation are given in the appendix. It is assumed that mixed fields of dislocations could be modeled by summing the combined distortion fields of edge and screw dislocations using the principle of superposition. When the bounds of integration are selected based on a simulated step size (with related implicit Burgers circuit) around a dislocation, these equations provide a simple solution to the problem of predicting the distortion field of a continuum array of dislocations of unit density as might be measured by EBSD.

When numerical derivatives of these simulated distortion fields were used to calculate $\alpha$ using Equation 2 , they were equivalent, within numerical error, to $\alpha$ calculated via Equation 1, demonstrating the validity of this method. The numerical results were also tested extensively against Green's function techniques (such as those presented in Mura (Mura, 1963, 1982)). It is important to note that this model for strain fields around continuum dislocation fields predicts strain gradients of comparable magnitude to rotation gradients because the infinitesimal dislocations are regularly arrayed instead of arranged to minimize strain.

\section{Results}

\subsection{Performance on simulated data}

In order to test the 3 and 6 constraint implementations of the Nye-Kröner-Bilby method of dislocation density calculation described in Section 3.1, lattice distortion fields for simulated volume elements were 
generated with known dislocation content. Measurable (via EBSD) distortion derivatives were calculated using the method described in Section 3.2 (the distortion was calculated at two points on the simulated surface, and the derivative taken numerically). This information was fed into each of implementations of Equation 3 to recover the detectable dislocation density in order to test the ability of each implementation to distinguish arbitrary dislocation types. The simulated material, nickel, was selected to be compatible with the experimental data set described in the next section. A single dislocation type with a density of $10^{15} \mathrm{~m} / \mathrm{m}^{3}$ was simulated in each interaction volume. The simulated regions of interest were cubes with side dimension of $100 \mathrm{~nm}$. The simulated lattice was oriented such that the $\left(\begin{array}{lll}1 & 1 & 0\end{array}\right)$ face of the crystal was parallel to the simulated surface and the y-direction corresponded to the [001] crystallographic direction (the same orientation as the single crystal examined in the next section). Eighteen different simulations were performed to show the detectability of each dislocation type given the sample's orientation. The resultant dislocation field calculated by each method is given in Figure 1.

The Nye-Kröner-Bilby method never completely matched the simulated dislocation densities for every simulation in either of its implementations (a perfect match would be indicated by the predicted dislocation density being identical to the simulated dislocation densities in Figure 1a). Ignoring the dislocation type and looking purely at the magnitude of the dislocation density, the 3 constraint implementation on average predicted $59 \%$ of the magnitude of the simulated dislocation content, but this ratio varied drastically depending on the orientation of the dislocations relative to the surface. Three constraints provided enough information to identify the correct dominant dislocation type in 5 out of 18 cases, and in two cases the predicted dislocation density was evenly divided between the simulated dislocation density and another erroneous dislocation type. The effect of using Pantleon's 6 constraints instead of just 3 is dramatic. The 6 constraint implementation detected on average $80 \%$ of the magnitude of the simulated dislocation density, and was able to correctly detect the dominant dislocation type in 16 of 18 cases, a $89 \%$ success rate. In the two cases where the dominant dislocation type was not detected, this was because at this specific orientation, these dislocation types are invisible to surface techniques. At an arbitrary orientation, the 6 constraint implementation can often detect all 18 dislocation types easily. The 6 constraint implementation occasionally predicts erroneous noise on other dislocation types, but this noise is relatively small. These simulations suggest that surface EBSD techniques can adequately measure arbitrary deformation when 6 constraints are used as opposed to 3 .

The validity of the 6 constraint implementation relies on the assumption that strain is negligible; otherwise only the 3 term method should be strictly correct. Because the simulations smear the dislocation density by evenly distributing the infinitesimal dislocations, strain is not minimized in the simulations as it would be in nature, leading to strain gradients with similar magnitude to the rotation gradients. As such, we may think of the simulations as a worst case scenario for the assumption that strain is negligible. Considering the excellent agreement of the 6 constraint implementation with the simulated densities, ignoring strain is clearly a viable assumption.

\subsection{Performance on a wedge-indented single crystal nickel specimen}

In this section we evaluate our selected implementation of the Nye-Kröner-Bilby method using a real sample. In a previous study, Kysar et al. examined the active dislocation types of a nickel single crystal that underwent a carefully controlled plastic deformation process (wedge indentation) (Kysar et al., 2010). The sample was deformed using a tungsten carbide wedge indenter with a $90^{\circ}$ included angle on the $\left(\begin{array}{lll}0 & 0 & 1\end{array}\right)$ face of a nickel single crystal. The indenter was oriented with the leading edge parallel to the $\left[\begin{array}{lll}1 & 1 & 0\end{array}\right]$ direction. The sample was then sectioned parallel to the $\left(\begin{array}{lll}1 & 1 & 0\end{array}\right)$ plane to reveal the portion of the sample that experienced plane strain. The sample was then polished and a $0.15 \mathrm{~mm} \times 0.15 \mathrm{~mm}$ area around the deformation was scanned using EBSD with a step size of 3 microns. This step size is large enough that it 
minimizes noise effects and the effects of SSDs, but small enough to resolve the visible slip bands. Because of the implications of plasticity around wedge indentations for fracture mechanics, this problem has been well studied (Crone et al., 2004; Rice, 1987; Kysar et al., 2007). By ensuring that the deformation was in-plane, Kysar, et. al. showed that only two components of the Nye tensor will be non-zero, $\alpha_{13}$ and $\alpha_{23}$. They also demonstrated that only three effective dislocation types could be activated, thus greatly simplifying the solution of Equation 3. Because of the extensive analysis of this highly constrained problem (including crystal plasticity validation (Saito and Kysar, 2010; Saito et al., 2012), we consider Kysar's results to provide an accurate baseline to verify the capability of the general implementation we evaluated in the previous section.

For convenience, Kysar's method of resolving dislocation density of individual dislocation types will be summarized here. Previous work has shown that a line load applied parallel to the $\left[\begin{array}{lll}1 & 1 & 0\end{array}\right]$ direction in an FCC crystal activates certain pairs of edge dislocation types in equal proportion to accommodate plane strain conditions, assuming the critically resolved shear stress for all dislocation types is equivalent. These pairs of equally activated dislocation types may be grouped together into three effective dislocation types of edge character. These dislocation types are listed in Table 1. Dislocation types are most properly called out in terms of the Burgers vector and dislocation line vector, but for easy relationship to well known crystallographic notation, they are presented here in terms of a slip direction and slip plane. For edge dislocation types, the dislocation line vector may be determined by taking the cross product of these two vectors. Because the deformation is carefully controlled to ensure plane strain conditions, only two terms of the Nye tensor are non-zero, $\alpha_{13}$ and $\alpha_{23}$. The other zero terms of the Nye tensor serve to constrain dislocation activity to occur only on the effective dislocation types, but do not provide any additional information as to the relative amount of activity on each of them. Thus, with three unknowns (the densities of the three effective dislocation types) and two constraints (associated with $\alpha_{13}$ and $\alpha_{23}$ ), solution of Equation 3 becomes trivial, and may be accomplished simply by examining the piece-wise objective function. The differences between Kysar's implementation of the Nye-Kröner-Bilby method and the implementation here is summarized in Table 2.

Table 1: Effective dislocation types required to accommodate plane strain in the $\left(\begin{array}{lll}1 & 1 & 0\end{array}\right)$ plane of an FCC crystal due to a line load parallel to the $\left[\begin{array}{lll}1 & 1 & 0\end{array}\right]$ direction (Kysar et al., 2010).

\begin{tabular}{|l|c|c|}
\hline$\#$ & Effective Burgers vector and slip plane & $\begin{array}{c}\text { Component crystallographic Burgers } \\
\text { vectors and slip planes }\end{array}$ \\
\hline 1 & $\left(\begin{array}{lll}1 & \overline{1} & 1\end{array}\right)\left[\begin{array}{lll}1 & 1 & 2\end{array}\right]$ & $\left(\begin{array}{lll}1 & \overline{1} & 1\end{array}\right)\left[\begin{array}{lll}1 & 0 & 1\end{array}\right],\left(\begin{array}{lll}1 & \overline{1} & 1\end{array}\right)\left[\begin{array}{lll}0 & 1 & 1\end{array}\right]$ \\
\hline 2 & $\left(\begin{array}{lll}0 & 0 & 1\end{array}\right)\left[\begin{array}{lll}1 & 1 & 0\end{array}\right]$ & $\left(\begin{array}{lll}1 & 1 & 1\end{array}\right)\left[\begin{array}{lll}1 & 1 & 0\end{array}\right],\left(\begin{array}{lll}\overline{1} & \overline{1} & 1\end{array}\right)\left[\begin{array}{lll}1 & 1 & 0\end{array}\right]$ \\
\hline 3 & $\left(\begin{array}{llll}1 & \overline{1} & \overline{1}\end{array}\right)\left[\begin{array}{lll}1 & \overline{1} & 2\end{array}\right]$ & $\left(\begin{array}{llll}1 & \overline{1} & \overline{1}\end{array}\right)\left[\begin{array}{llll}1 & 0 & 1\end{array}\right],\left(\begin{array}{lll}1 & \overline{1} & \overline{1}\end{array}\right)\left[\begin{array}{lll}0 & \overline{1} & 1\end{array}\right]$ \\
\hline
\end{tabular}

In this work, the Nye-Kröner-Bilby method (Equation 3) was implemented as described in the previous section, and as summarized in Table 2 and applied to Kysar's original EBSD data. HREBSD was used to calculate the lattice distortion gradients on the sample surface and subsequently the five terms and one difference of terms recoverable from the Nye tensor. All 18 crystallographic dislocation types were considered, making no assumptions about which types would be active. The tolerances on the constraints were relaxed to allow for noise of magnitude, $\Delta \theta / L$, where $L$ is the step size of the EBSD scan and $\Delta \theta$ is the estimated error of the HREBSD technique, which Wilkinson quotes as being on the order of 100 microstrain (Wilkinson et al., 2006a). The calculated dislocation density for each of the 18 dislocation types are shown 
Table 2: A comparison between the implementation of the Nye-Kröner-Bilby method here and in the work of Kysar et al. (2007, 2010).

\begin{tabular}{|c|c|c|}
\hline & Kysar's implementation & General 6 constraint implementation \\
\hline Constraints & $\alpha_{13}$ and $\alpha_{23}$ & $\alpha_{13}, \alpha_{23}, \alpha_{33}, \alpha_{12}, \alpha_{21}$ and $\alpha_{11}-\alpha_{22}$ \\
\hline $\begin{array}{l}\text { Dislocation } \\
\text { types }\end{array}$ & 3 effective types & 18 crystallographic types \\
\hline $\begin{array}{l}\text { Optimization } \\
\text { method }\end{array}$ & Inspection of the objective function & Linear programming \\
\hline $\begin{array}{l}\text { Gradient } \\
\text { calculation }\end{array}$ & Hough based, no strain & HREBSD \\
\hline Advantages & No ambiguity in the solution & $\begin{array}{c}\text { Independent of } \\
\text { deformation/orientation }\end{array}$ \\
\hline Disadvantages & $\begin{array}{l}\text { Tailored for a specific deformation } \\
\text { case/orientation }\end{array}$ & $\begin{array}{l}\text { Some noise, relies on the minimum } \\
\text { energy assumption }\end{array}$ \\
\hline
\end{tabular}

in Figure 2. For easier comparison with the previous study's results, the dislocation density on the effective dislocation types defined by Kysar were calculated by summing the density on each of their component crystallographic types. These results are shown in Figures 3-5. The dislocation density maps were locally averaged to reduce apparent noise in the data.

The three effective dislocation types were all clearly detected, and the dislocation density fields closely match previous microscopy and finite element work for this sample. Effective dislocation types 1 and 3 were antisymmetric to each other about the axis of the leading edge of the indenter (dislocation type 1 dominant to the right of the indenter and type 3 to the left), while effective dislocation type 2 is symmetric about it. All other dislocation types have values below the noise level, with the possible exception of some screw types. The mean total dislocation density for the scanned area is $2.41 \times 10^{13} \mathrm{~m} / \mathrm{m}^{3}$. The results show good agreement with Kysar's implementation of the Nye-Kröner-Bilby method, but the general implementation presented here has the advantage that it requires no prior knowledge of the deformation of the sample. This general implementation of the Nye-Kröner-Bilby method is just as effective at identifying both magnitude and type of dislocations due to planar deformation as a highly constrained method, as has already been suggested by simulation.

\section{Conclusions}

Numerical simulations were used to validate the ability of a general implementation of the Nye-KrönerBilby method for calculating dislocation density on individual dislocation types from measured HREBSD data. The simulations suggested that when 6 constraints from the Nye tensor are employed as suggested by Pantleon, surface HREBSD techniques are able to detect almost any dislocation content of a material 
without the need for serial sectioning and without making any assumptions about the deformation of the sample. Only very specifically oriented dislocations avoid detection.

A previously analyzed dislocation field in wedge-indented single crystal nickel from a study by Kysar et al. was used to demonstrate the application of the Nye-Kröner-Bilby method, as implemented here. Results closely matched the previous study which was validated by an analysis of the deformation mechanics. The method described in this paper has three advantages over Kysar's implementation, namely (i) it may be applied generally without assuming the active dislocation types, (ii) it captures some dislocation information associated with out of plane deformation due to the inclusion of 3 additional constraints, and (iii) it recovers more degrees of freedom in determining the active dislocation types.

\section{Acknowledgments}

This work was supported by National Science Foundation grant CMMI 1404771 and Department of Energy grant DE-SC0012587 as well as additional funding from EDAX. We would also like to acknowledge a number of helpful discussions with Stuart Wright of EDAX/TSL.

\section{Appendix}

In Section 3.2, we defined the $\beta_{x x}$ component of the "smeared" lattice distortion of an idealized edge dislocation at a point, $\left(x_{0}, y_{0}\right)$ as:

$$
\beta_{x x}\left(x_{0}, y_{0}\right)=-\frac{b}{4 \pi(1-v)(t-s)(q-p)} \int_{y=p}^{q} \int_{x=s}^{t} \frac{y_{0}-y}{r_{0}^{2}}\left((1-2 v)+\frac{2\left(x_{0}-x\right)^{2}}{r_{0}^{2}}\right) d x d y
$$

where $r_{0}^{2}=\left(x_{0}-x\right)^{2}+\left(y_{0}-y\right)^{2}$. To solve this equation, the following substitutions are convenient:

$$
\begin{aligned}
& Q\left(x_{0}, y_{0}, q, p, t, s\right)=\int_{y=p}^{q} \int_{x=s}^{t} \frac{y_{0}-y}{r_{0}^{2}} d x d y \\
&=I\left(x_{0}, y_{0}, q, t\right)-I\left(x_{0}, y_{0}, q, s\right)-I\left(x_{0}, y_{0}, p, t\right)+I\left(x_{0}, y_{0}, p, s\right)
\end{aligned}
$$

where $I\left(x_{0}, y_{0}, q, t\right)=\left(x_{0}-t\right) \frac{\ln \left(\left(y_{0}-q\right)^{2}+\left(x_{0}-t\right)^{2}\right)}{2}-\left(y_{0}-q\right) \tan ^{-1}\left(-\frac{\left(x_{0}-t\right)}{\left(y_{0}-q\right)}\right)+t$ and

$$
\begin{aligned}
R\left(x_{0}, y_{0}, q, p, t, s\right)=\int_{y=p}^{q} \int_{x=s}^{t} \frac{\left(y_{0}-y\right)\left(x_{0}-x\right)^{2}}{r_{0}^{4}} d x d y \\
\quad=J\left(x_{0}, y_{0}, q, t\right)-J\left(x_{0}, y_{0}, q, s\right)-J\left(x_{0}, y_{0}, p, t\right)+J\left(x_{0}, y_{0}, p, s\right)
\end{aligned}
$$

where $J\left(x_{0}, y_{0}, q, t\right)=-\frac{\left(y_{0}-q\right)}{2} \tan ^{-1}\left(-\frac{\left(x_{0}-t\right)}{\left(y_{0}-q\right)}\right)+\frac{t}{2}$. Substituting in Equations 16 and 17, Equation 15 becomes:

$$
\beta_{x x}\left(x_{0}, y_{0}\right)=-\frac{b}{4 \pi(1-v)(t-s)(q-p)}\left[(1-2 v) Q\left(x_{0}, y_{0}, q, p, t, s\right)+2 R\left(x_{0}, y_{0}, q, p, t, s\right)\right]
$$


Similarly for the other non-zero components of lattice distortion around a smeared edge dislocation:

$$
\begin{aligned}
& \beta_{x y}\left(x_{0}, y_{0}\right)=\frac{b}{4 \pi(1-v)(t-s)(q-p)}\left[(3-2 v) Q\left(y_{0}, x_{0}, q, p, t, s\right)-2 R\left(y_{0}, x_{0}, q, p, t, s\right)\right] \\
& \beta_{y x}\left(x_{0}, y_{0}\right)=-\frac{b}{4 \pi(1-v)(t-s)(q-p)}\left[(1-2 v) Q\left(y_{0}, x_{0}, q, p, t, s\right)+2 R\left(y_{0}, x_{0}, q, p, t, s\right)\right] \\
& \beta_{y y}\left(x_{0}, y_{0}\right)=-\frac{b}{4 \pi(1-v)(t-s)(q-p)}\left[(1-2 v) Q\left(x_{0}, y_{0}, q, p, t, s\right)-2 R\left(x_{0}, y_{0}, q, p, t, s\right)\right]
\end{aligned}
$$

Likewise for a screw dislocation:

$$
\begin{aligned}
& \beta_{z x}\left(x_{0}, y_{0}\right)=-\frac{b}{2 \pi(t-s)(q-p)} Q\left(x_{0}, y_{0}, q, p, t, s\right) \\
& \beta_{z y}\left(x_{0}, y_{0}\right)=-\frac{b}{2 \pi(t-s)(q-p)} Q\left(y_{0}, x_{0}, q, p, t, s\right)
\end{aligned}
$$

\section{References}

Adams, B. L., 1997. Orientation imaging microscopy: Emerging and future applications. Ultramicroscopy 67 (1-4), 11-17.

Arsenlis, A., Parks, D. M., 1999. Crystallographic aspects of geometrically-necessary and statistically-stored dislocation density. Acta Materialia 47 (5), 1597-1611.

Ashby, M. F., 1970. The deformation of plastically non-homogeneous materials. Philosophy Magazine 21, $399-424$.

Baitsch, M., Le, K., Tran, T., 2015. Dislocation structure during microindentation. International Journal of Engineering Science 94, $195-211$.

Bardella, L., Giacomini, A., 2008. Influence of material parameters and crystallography on the size effects describable by means of strain gradient plasticity. Journal of the Mechanics and Physics of Solids 56, 2906-2934.

Basinger, J., Fullwood, D., Kacher, J., Adams, B., 2011. Pattern center determination in EBSD microscopy. Microscopy and Microanalysis 17, 330-340.

Bilby, B., Gardner, L., Smith, E., 1958. The relation between dislocation density and stress. Acta Metallurgica 6 (1), 29 - 33.

Britton, T., Maurice, C., Fortunier, R., Driver, J., Day, A., Meaden, G., Dingley, D., Mingard, K., Wilkinson, A., 2010. Factors affecting the accuracy of high resolution electron backscatter diffraction when using simulated patterns. Ultramicroscopy 110 , $1443-1453$.

Britton, T. B., Wilkinson, A. J., 2012. Stress fields and geometrically necessary dislocation density distributions near the head of a blocked slip band. Acta Materialia 60 (16), 5773 - 5782.

Calcagnotto, M., Ponge, D., Demir, E., Raabe, D., 2010. Orientation gradients and geometrically necessary dislocations in ultrafine grained dual phase steels studied by 2D and 3D EBSD. Materials Science and Engineering A 527, 2738-2746.

Crone, W., Shield, T., Creuziger, A., Henneman, B., 2004. Orientation dependence of the plastic slip near notches in ductile FCC single crystals. Journal of the Mechanics and Physics of Solids 52 (1).

Demir, E., Raabe, D., Zaafarani, N., Zaefferer, S., 2009. Investigation of the indentation size effect through the measurement of the geometrically necessary dislocations beneath small indents of different depths using EBSD tomography. Acta Materialia 57, 559-569.

El-Dasher, B., Adams, B., Rollett, A., 2003. Viewpoint: Experimental recovery of geometrically necessary dislocation density in polycrystals. Scripta Materialia 48 (2), 141-145.

Evers, L., Brekelmans, W., Geers, M., 2004. Scale dependent crystal plasticity framework with dislocation density and grain boundary effects. International Journal of Solids and Structures 41, 5209-5230.

Field, D., Magid, K., Mastorakos, I., Florando, J., Lassila, D., Jr., J. M., 2010. Mesoscale strain measurement in deformed crystals: A comparison of x-ray microdiffraction with electron backscatter diffraction. Philosophical Magazine 90 (11), 1451-1464.

Fleck, N., Hutchinson, J., 1997. Strain gradient plasticity. Advances in Applied Mechanics 33, 296-358.

Fullwood, D., Vaudin, M., Daniels, C., Ruggles, T., Wright, S. I., 2015. Validation of kinematically simulated pattern HR-EBSD for measuring absolute strains and lattice tetragonality. Materials Characterization 107, 270 - 277.

Gardner, C. J., Adams, B. L., Basinger, J., Fullwood, D. T., 2010. EBSD-based continuum dislocation microscopy. International Journal of Plasticity 26, 1234-1247. 
Hardin, T., Ruggles, T., Koch, D., Niezgoda, S., Fullwood, D., Homer, E., 2015. Analysis of traction-free assumption in highresolution EBSD measurements. Journal of Microscopy.

URL http://dx.doi.org/10.1111/jmi.12268

Hardin, T. J., Adams, B. L., Fullwood, D. T., Wagoner, R. H., Homer, E. R., 2013. Estimation of the full Nye's tensor and its gradients by micro-mechanical stereo-inference using EBSD dislocation microscopy. International Journal of Plasticity 50, 146 $-157$.

Hirsch, P. B., Howie, A., Whelan, M. J., 1960. A kinematical theory of diffraction contrast of electron transmission microscope images of dislocations and other defects. Philosophical Transactions of the Royal Society of London A: Mathematical, Physical and Engineering Sciences 252 (1017), 499-529.

Jiang, J., Britton, T. B., Wilkinson, A. J., 2015. The orientation and strain dependence of dislocation structure evolution in monotonically deformed polycrystalline copper. International Journal of Plasticity 69, 102-117.

Kacher, J., Landon, C., Adams, B. L., Fullwood, D., 2009. Braggs law diffraction simulations for electron backscatter diffraction analysis. Ultramicroscopy 109 (9), 1148-1156.

Karamched, P. S., Wilkinson, A. J., 2011. High resolution electron back-scatter diffraction analysis of thermally and mechanically induced strains near carbide inclusions in a superalloy. Acta Materialia 59 (1), $263-272$.

Khosravani, A., Scott, J., Miles, M., Fullwood, D., Adams, B., Mishra, R., 2013. Twinning in magnesium alloy AZ31B under different strain paths at moderately elevated temperatures. International Journal of Plasticity 45 (0), 160-173, in Honor of Rob Wagoner.

Koike, J., Kobayashi, T., Mukai, T., Watanabe, H., Suzuki, M., Maruyama, K., Higashi, K., 2003. The activity of non-basal slip systems and dynamic recovery at room temperature in fine-grained AZ31b magnesium alloys. Acta Materialia 51, $2055-65$.

Kröner, E., 1958. Continuum theory of dislocations and self-stresses. Ergebnisse der Angewandten Mathematik 5, 1327-1347.

Kubin, L., Mortenson, A., 2003. Geometrically necessary dislocations and strain-gradient plasticity: a few critical issues. Scripta Materialia 48, 119-125.

Kysar, J., Saito, Y., Oztop, M., Lee, D., Huh, W., 2010. Experimental lower bounds on geometrically necessary dislocation density. International Journal of Plasticity 26, 1097-1123.

Kysar, J. W., Gan, Y., Morse, T., Chen, X., Jones, M., 2007. High strain gradient plasticity associated with wedge indentation into face-centered cubic single crystals: Geometrically necessary dislocation densities. Journal of the Mechanics and Physics of Solids 55, 1554-1573.

Lazar, M., 2003. Dislocations in the field theory of elastoplasticity. Computational Materials Science 28, 419-428.

Lim, H., Subedi, S., Fullwood, D., Adams, B., Wagoner, R., 2014. A practical meso-scale polycrystal model to predict dislocation densities and the Hall-Petch effect. Materials Transactions 55 (1), 35-38.

Littlewood, P., Britton, T., Wilkinson, A., 2011. Geometrically necessary dislocation density distribution in Ti-6Al-4V deformed in tension. Acta Materialia 59, 6489-6500.

Ma, A., Roters, F., Raabe, D., 2006. On the consideration of interactions between dislocations and grain boundaries in crystal plasticity finite element modeling Theory, experiments, and simulations. Acta Materialia 54 (8), 2181 - 2194.

Maurice, C., Krzysztof, D., Fortunier, R., 2011. A method for accurate localisation of EBSD pattern centres. Ultramicroscopy 111, $140-148$.

Mura, T., 1963. Continuous distribution of moving dislocations. Philosophical Magazine 8, 843-857.

Mura, T., 1982. Micromechanics of Defects in Solids, 1st Edition. Martinus Nijhoff Publishers, Dordrecht.

Nye, J., 1953. Some geometrical relations in dislocated crystals. Acta Metallurgica 1, 153-162.

Pantleon, W., 2008. Resolving the goemetrically necessary dislocation content by conventional electron backscattering diffraction. Scripta Materialia 58, 994-997.

Rice, J. R., 1987. Tensile crack tip fields in elastic-ideally plastic crystals. Mechanics of Materials 6, 317-335.

Ruggles, T. J., Fullwood, D. T., 2013. Estimations of bulk geometrically necessary dislocation density using high resolution EBSD. Ultramicroscopy 133, 8-15.

Saito, Y., Kysar, J. W., 2010. Wedge indentation into elastic-plastic single crystals, 1: Asymptotic fields for nearly-flat wedge. International Journal of Plasticity 27, 1640-1657.

Saito, Y., Oztop, M. S., Kysar, J. W., 2012. Wedge indentation into elastic-plastic single crystals. 2: Simulations for face-centered cubic crystals. International Journal of Plasticity 28, 70-87.

Schwartz, A., Kumar, M., Adams, B., Field, D., 2009. Electron backscatter diffraction in materials science, 2nd Edition. Springer, New York.

Sun, S., Adams, B., King, W., 2000. Observations of lattice curvature near the interface of a deformed aluminium bicrystal. Philosophical Magazine A: Physics of Condensed Matter, Structure, Defects and Mechanical Properties 80 (1), 9-25.

Teodosiu, C., 1982. Elastic Models of Crystal Defects. Springer-Verlag, Berlin.

Troost, K., van der Sluis, P., Gravesteijn, D., 1993. Microscale elastic-strain determination by backscatter kikuchi diffraction in the scanning electron microscope. Appl. Phys. Lett. 62 (10), 1110-1112. 
Ungár, T., Langford, J., Cernik, R., Vörös, G., Pflaumer, R., Oszlányi, G., Kovács, I., 1998. Microbeam X-ray diffraction studies of structural properties of polycrystalline metals by means of synchrotron radiation. Materials Science and Engineering A 247, $81-87$.

Wheeler, J., Mariani, E., Piavzolo, S., Prior, D., Trimby, P., Drury, M., 2009. The weighted Burgers vector: a new quantity for constraining dislocation densities and types using electron backscatter diffraction on 2D sections through crystalline materials. Journal of Microscopy 233, 482-494.

Wilkinson, A., Clarke, E., Britton, T., Littlewood, P., Karamched, P., 2010. High-resolution electron backscatter diffraction: an emerging tool for studying local deformation. The Journal of Strain Analysis for Engineering Design 45, 365-376.

Wilkinson, A., Randman, D., 2010. Determination of elastic strain fields and geometrically necessary dislocation distributions near nanoindents using electron back scatter diffraction. Philosophical Magazine 90 (9), 1159-1177.

Wilkinson, A. J., Meaden, G., Dingley, D. J., 2006a. High-resolution elastic strain measurement from electron backscatter diffraction patterns: New levels of sensitivity. Ultramicroscopy 106, 307-313.

Wilkinson, A. J., Meaden, G., Dingley, D. J., 2006b. High resolution mapping of strains and rotations using electron back scatter diffraction. Materials Science and Technology 22 (11), 1-11.

Winkelmann, 2007. Simulation of electron backscatter diffraction patterns. Microscopy and Microanalysis 13, 930-931.

Zhang, T., Collins, D. M., Dunne, F. P., Shollock, B. A., 2014. Crystal plasticity and high-resolution electron backscatter diffraction analysis of full-field polycrystal Ni superalloy strains and rotations under thermal loading. Acta Materialia 80 (0), 25 - 38. 
Figure 1: The simulated (a) and predicted dislocation densities using 3 (b) and 6 (c) constraints in $\mathrm{m} / \mathrm{m}^{3}$. The horizontal axis corresponds to the distinct dislocation type (\#1-6 are screw dislocations, and \#7-18 are edge dislocations). The vertical axis relates to the simulation number (i.e. each row represents a simulated volume element), and the color scale refers to the dislocation density assigned to each dislocation type.

Figure 2: GND density maps for all 18 unique dislocation types as calculated by the 6-constraint Nye-Kröner-Bilby method. Edge dislocations are called out by slip plane and Burgers vector; screw dislocations are called out by Burgers vector alone. The boxed dislocation types are the dislocation types associated with Kysar's effective dislocation types. The scanned area is $750 \times 750$ microns.

Figure 3: Dislocation density maps for the (a) $\left(\begin{array}{lll}1 & \overline{1} & 1\end{array}\right)\left[\begin{array}{lll}1 & 0 & 1\end{array}\right]$ and (b) (ll $\left.\begin{array}{lll}1 & 1\end{array}\right)\left[\begin{array}{lll}0 & 1 & 1\end{array}\right]$ edge dislocation types, around an indented nickel single crystal as determined by the implementation of the Nye-Kröner-Bilby method presented here. For comparison with Kysar's method, these values are summed to show the dislocation density of effective dislocation type $1,\left(\begin{array}{lll}1 & 1 & 1\end{array}\right)\left[\begin{array}{lll}1 & 1 & 2\end{array}\right]$ (c). The scanned area is $750 \times 750$ microns.

Figure 4: Dislocation density maps for the (a) $\left(\begin{array}{lll}1 & 1 & 1\end{array}\right)\left[\begin{array}{lll}1 & 1 & 0\end{array}\right]$ and (b) $\left(\begin{array}{lll}1 & 1 & 1\end{array}\right)\left[\begin{array}{lll}1 & 1 & 0\end{array}\right]$ edge dislocation types, around an indented nickel single crystal as determined by the implementation of the Nye-Kröner-Bilby method presented here. For comparison with Kysar's method, these values are summed to show the dislocation density of effective dislocation type 2, $\left(\begin{array}{lll}0 & 0 & 1\end{array}\right)\left[\begin{array}{lll}1 & 1 & 0\end{array}\right]$ (c). The scanned area is $750 \times 750$ microns.

Figure 5: Dislocation density maps for the (a) $\left(\begin{array}{lll}1 & 1 & 1\end{array}\right)\left[\begin{array}{lll}1 & 0 & 1\end{array}\right]$ and (b) $\left(\begin{array}{lll}1 & 1 & 1\end{array}\right)\left[\begin{array}{lll}0 & \overline{1} & 1\end{array}\right]$ edge dislocation types, around an indented nickel single crystal as determined by the implementation of the Nye-Kröner-Bilby method presented here. For comparison with Kysar's method, these values are summed to show the dislocation density of effective dislocation type $3,\left(\begin{array}{lll}1 & \overline{1} & \overline{1}\end{array}\right)\left[\begin{array}{lll}1 & \overline{1} & 2\end{array}\right]$ (c). The scanned area is $750 \times 750$ microns. 

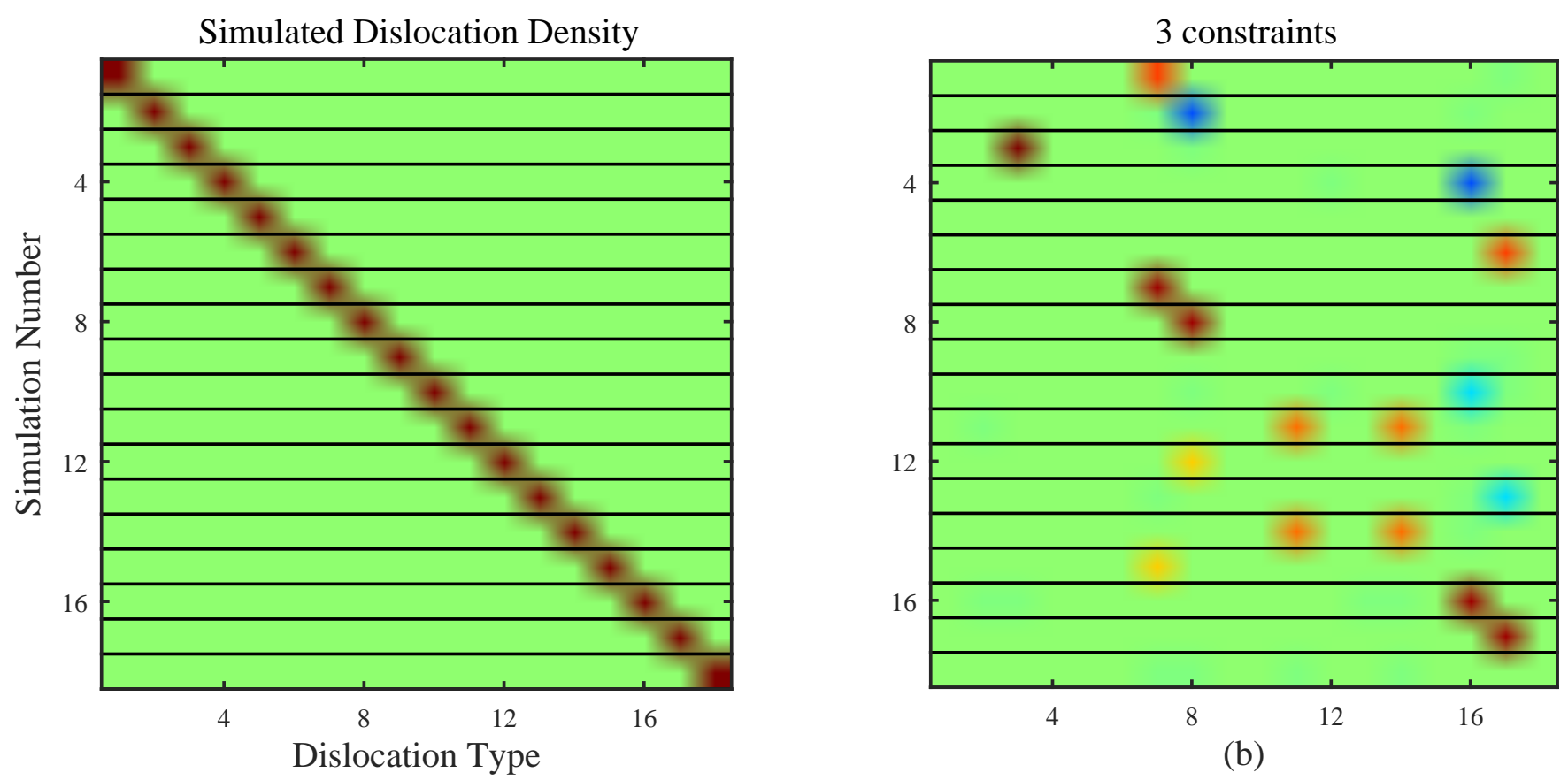

(a)

(b)

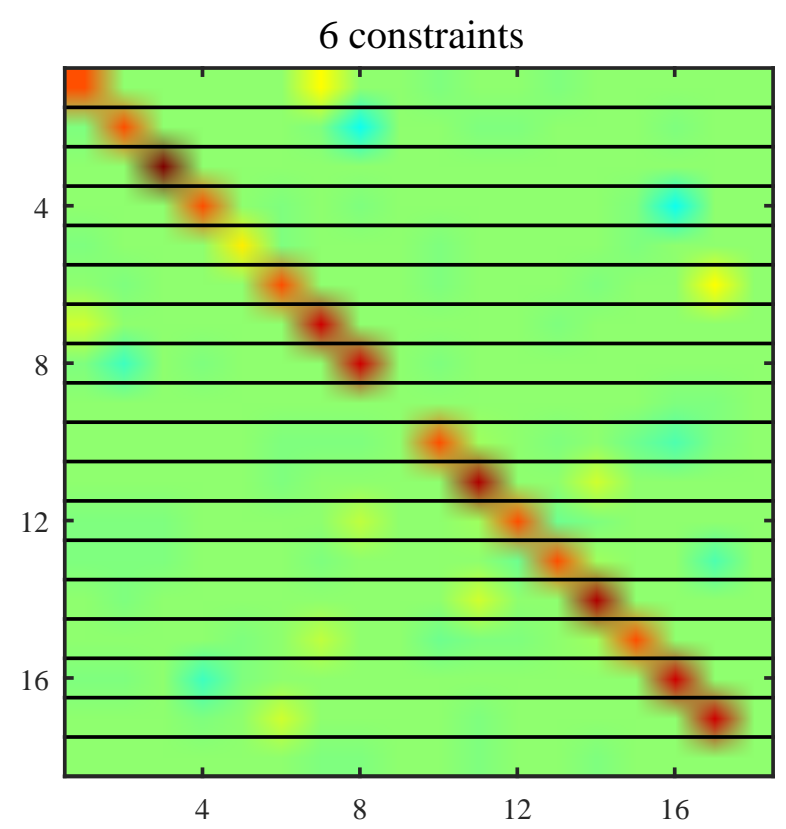

(d) 


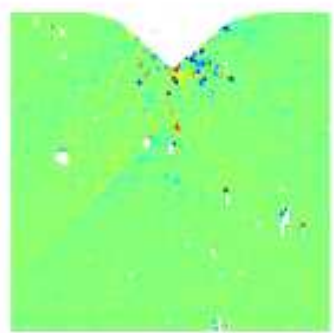

(screw)[101]

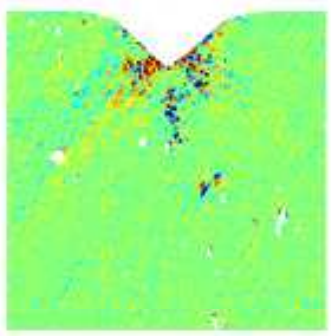

(screw)[1110]

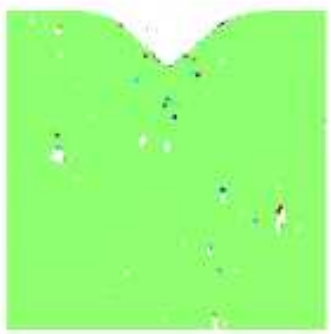

(11̄i) [110]

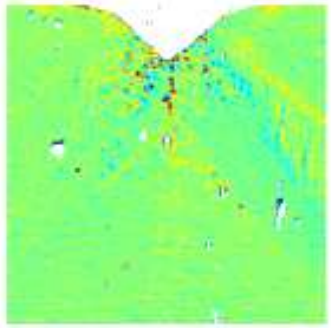

(1̄i1) [011]

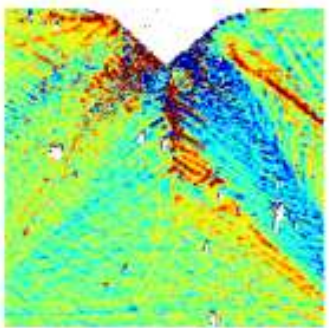

(1111) [011]

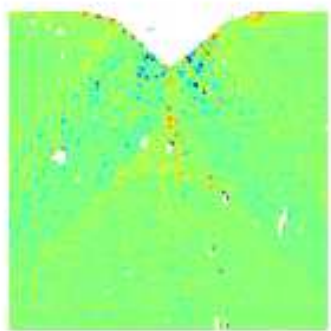

(screw) $[0 \overline{1} 1]$

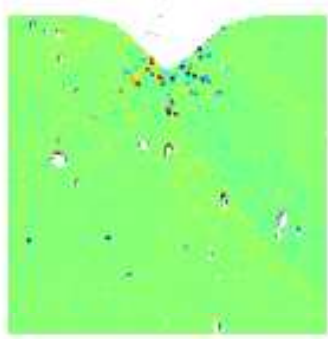

(screw)[011]

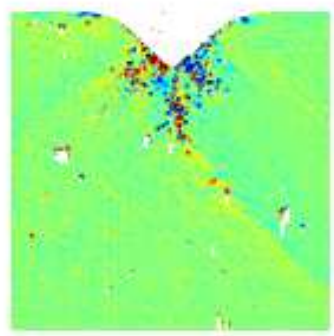

(111) [101]

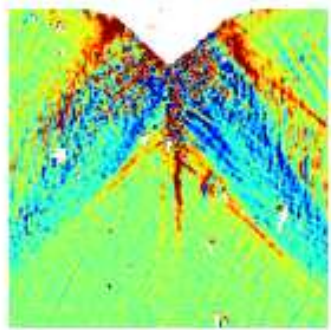

(111) [110]

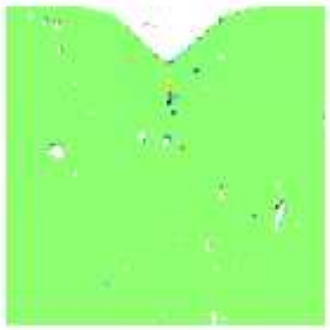

(1111) [110]

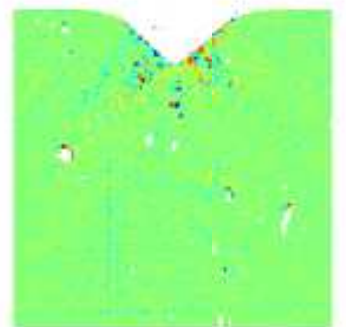

(screw)[110]

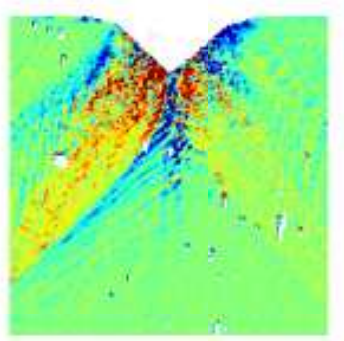

(11ī) [101]

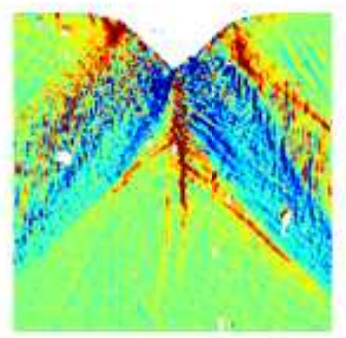

(111) [110]

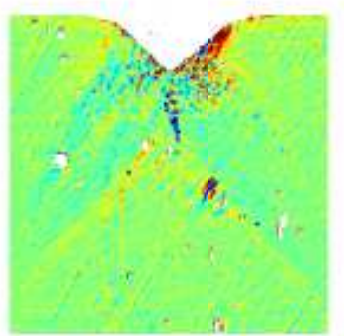

(1111)[101]

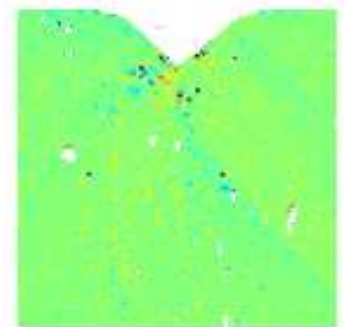

(screw)[101]

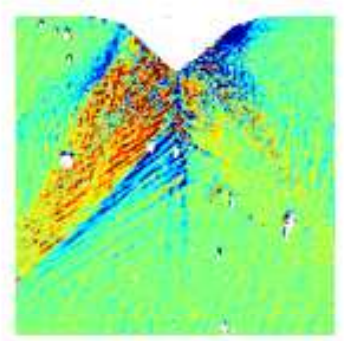

(11̄ī) $[0 \overline{1} 1]$

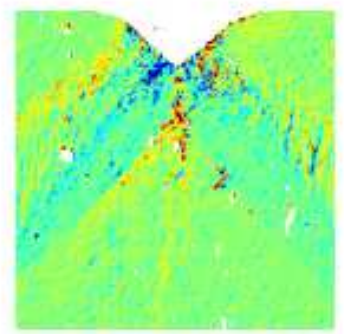

(111) [0̄1]

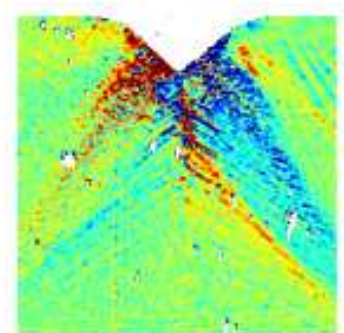

(111) [101]

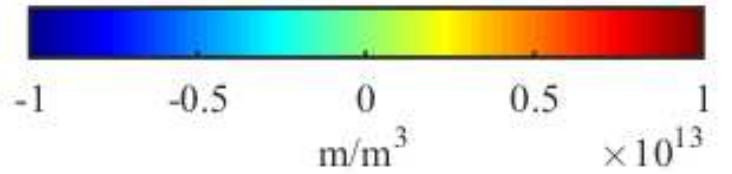




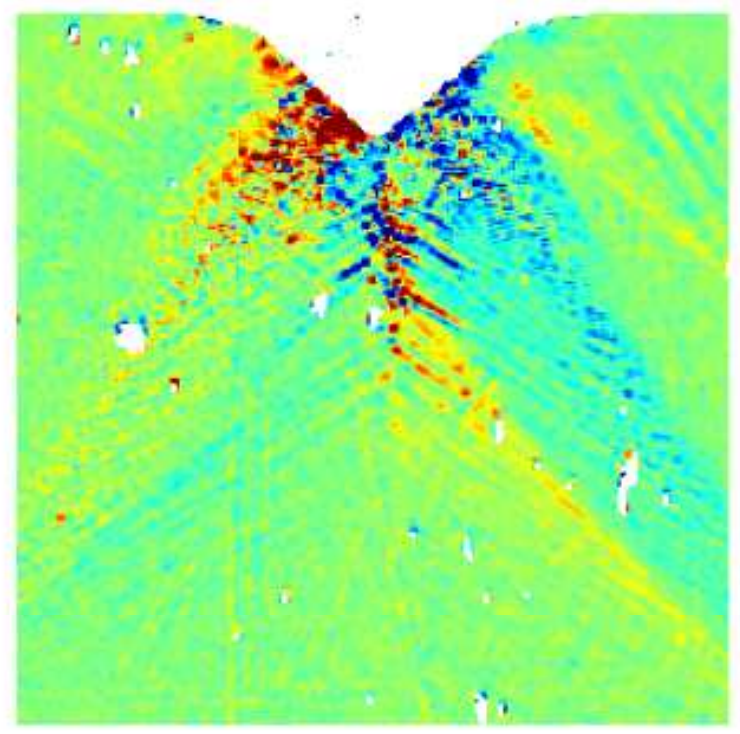

(a)

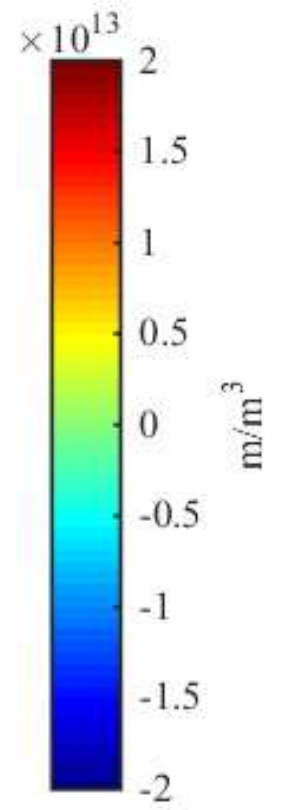

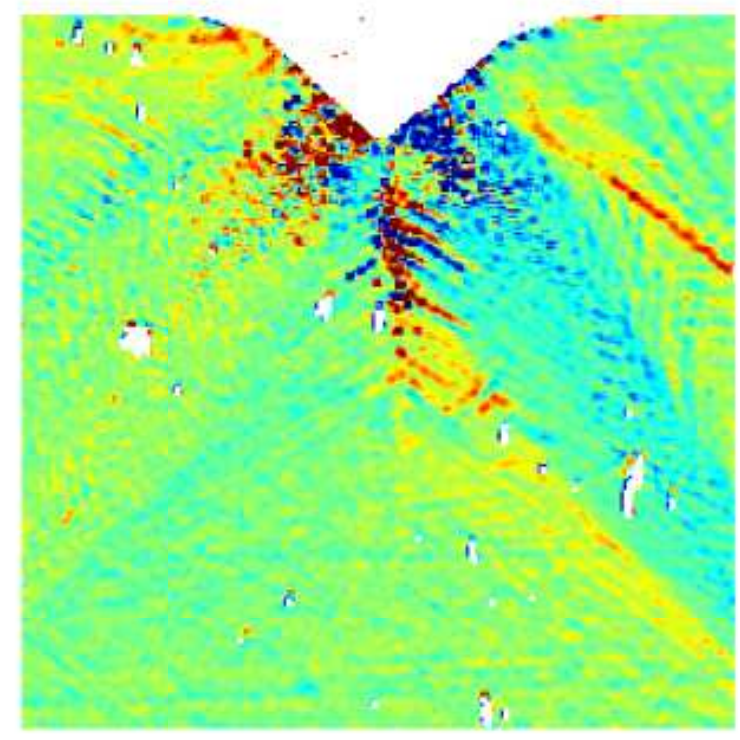

(b)

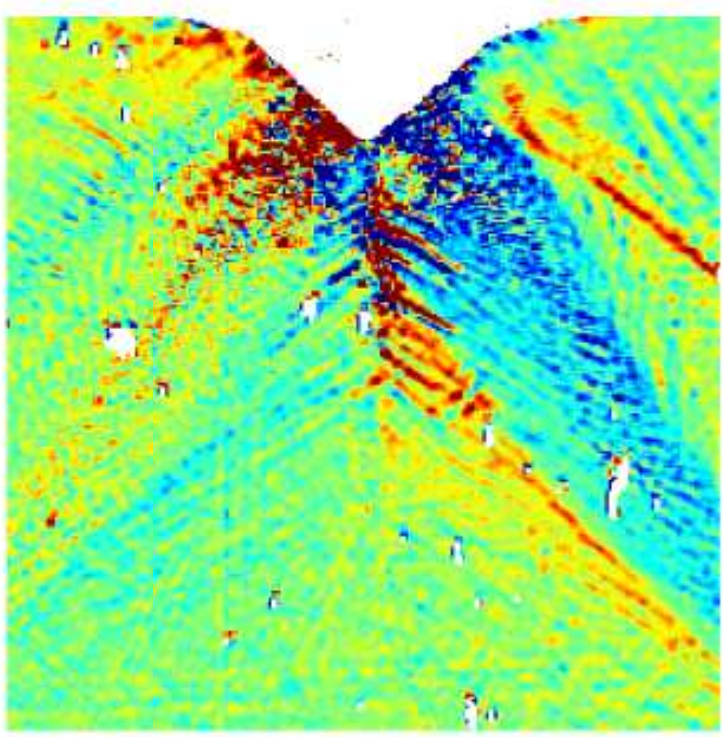

(c) 


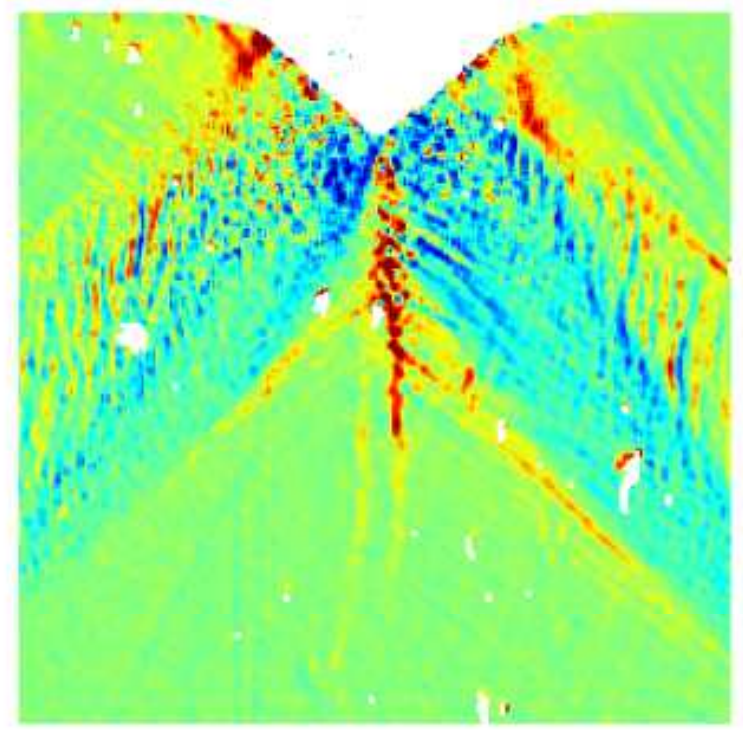

(a)

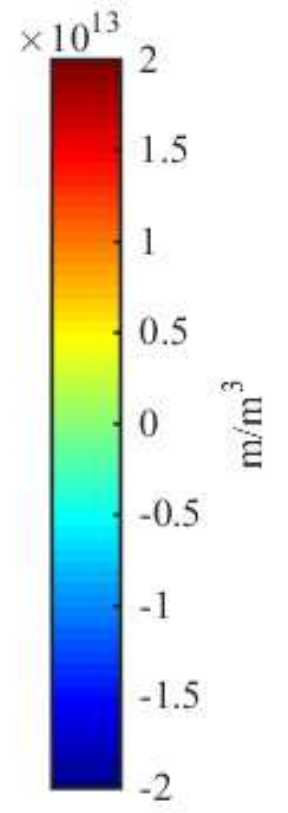

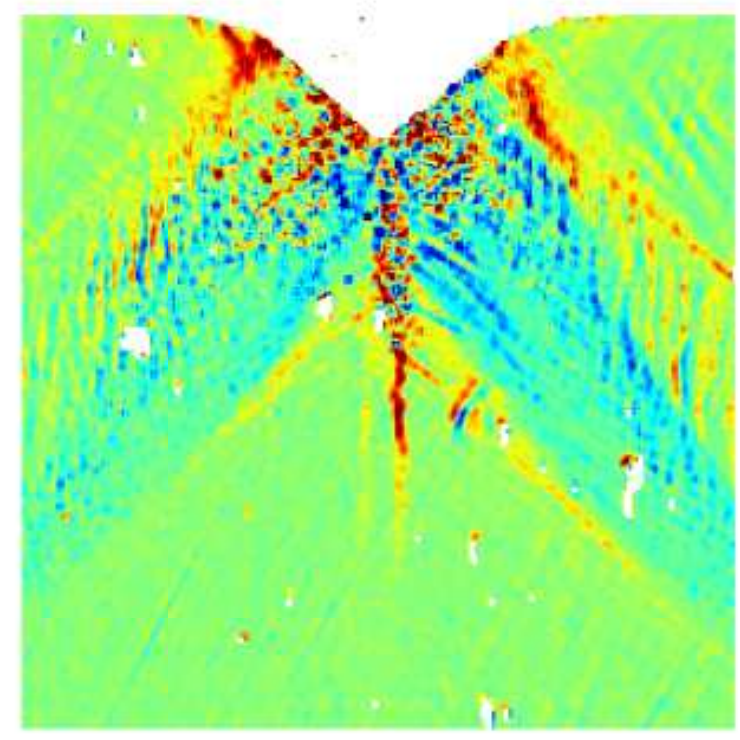

(b)

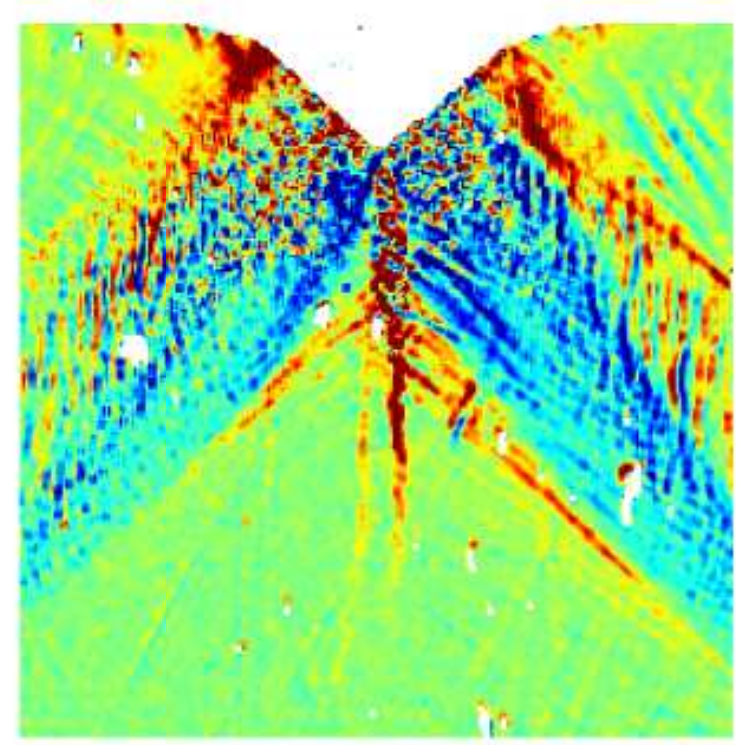

(c) 


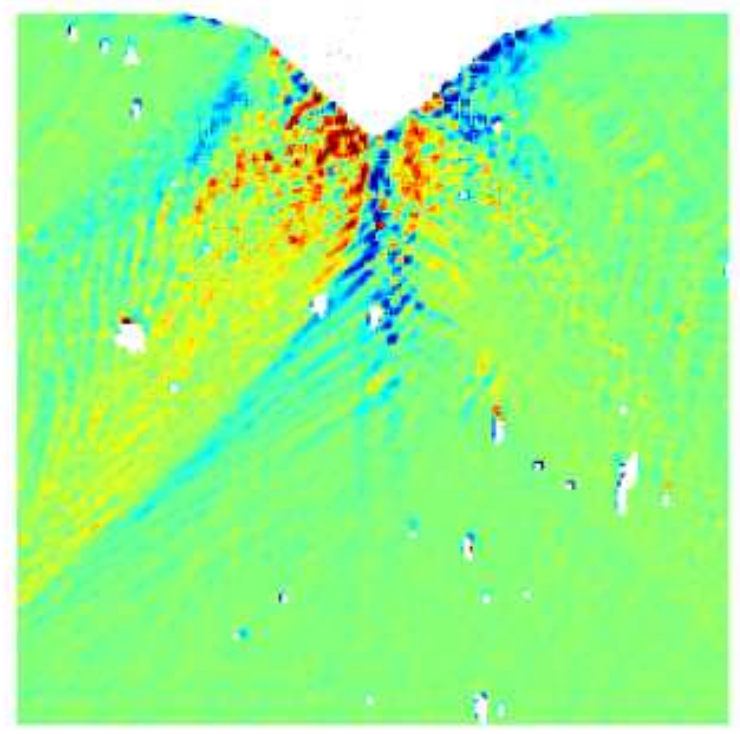

(a)

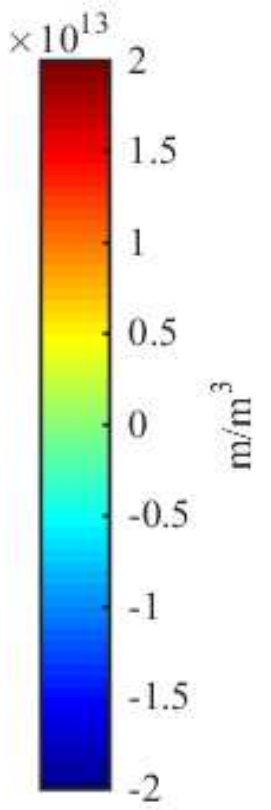

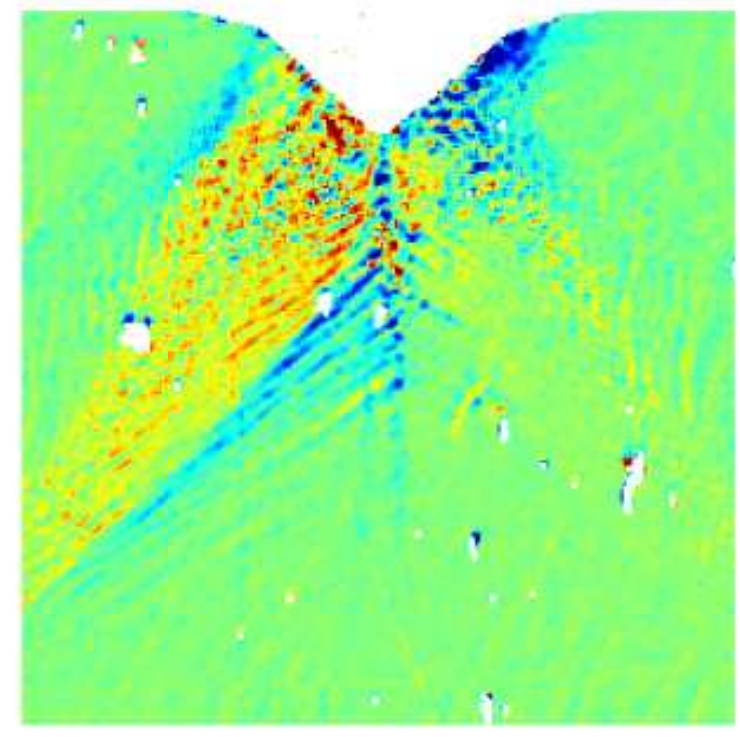

(b)

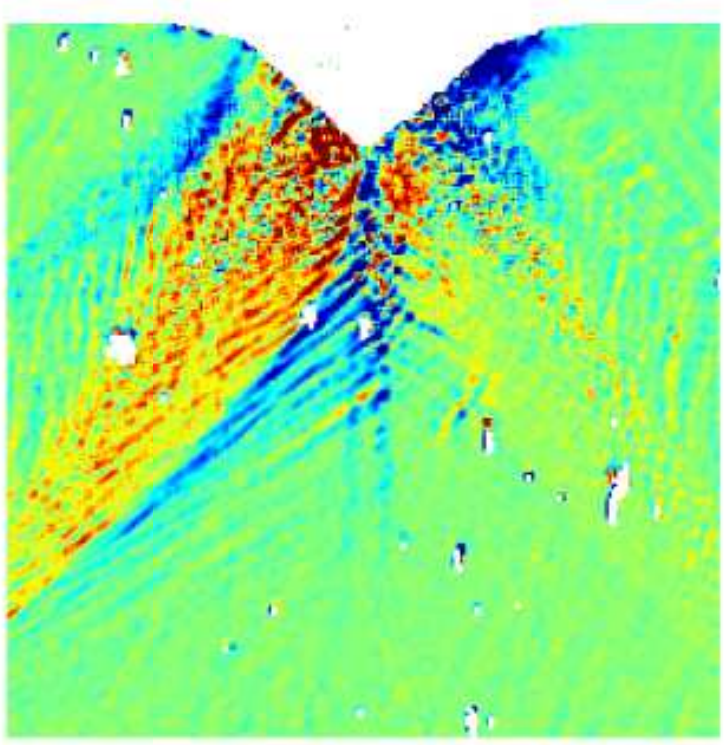

(c) 